\title{
The Performance of the Laser Systems in the Calibration System of LHAASO-WFCTA
}

\author{
N.Xie, Q.H. Chen, H. Liu, Y. He, W.J. Long, H.Y. Jia, F.R. Zhu* for the LHAASO \\ Collaboration ${ }^{\dagger}$ \\ School of Physical Science and Technology, Southwest Jiaotong University, Chengdu, 611756, \\ China \\ E-mail: zhufr@home.swjtu.edu.cn
}

\section{Y. Zhang}

Institute of High Energy Physics, Chinese Academy of Sciences, Beijing 100049, China

\begin{abstract}
Wide Field view of Cherenkov Telescope Array(WFCTA of LHAASO, consisting of 18 telesopes, has been proposed to mount at Daocheng County, Sichuan Province, China, to probe the galactic cosmic rays with the energies from $3 \times 10^{13} \mathrm{eV}-10^{1} 7 \mathrm{eV}$ together with WCDA and KM2A of LHAASO. 2 N2 laser systems and 2 YAG laser systems will be built to calibrate the detection effeciency of WFCTA and monitoring atmosphere. The laser beam in N2 laser systems, originally the rectangle facula with $3 \mathrm{~mm}$ times $7 \mathrm{~mm}$, are bound the circular facula with the radius not more than $10 \mathrm{~mm}$ at the $20 \mathrm{~cm}$ to the N2 laser head. The N2 laser systems are relatively simple to run normally in our testing, at LHAASO base and the campus of Southwest Jiaotong University. To keep a relatively constant temperature condition, an auto-heating belt is fixed outside of the YAG laser head. In this paper the performance of the 4 laser systems will be reported in detail.
\end{abstract}

36th International Cosmic Ray Conference -ICRC2019-

July 24th - August 1st, 2019

Madison, WI, U.S.A.

\footnotetext{
* Speaker.

$\dagger$ Supported by National Natural Science Foundation of China (11503021,11205126,11847307), Key Projects of Cooperation between Provincial Colleges and Schools of Science and Technology Plan from Sichuan Province(No. 2017JZ0034) and National Key Research and Development Program Projects(No. 2018YFA0404201)
} 


\section{Introduction}

The Large High Altitude Air Shower(LHAASO), one of the Major National Science and Technology Infrastructures, is located at Haizi Mountain, Daocheng County, Sichuan Province. It is composed of the Square Kilometer Array(KM2A), Water Cherenkov Detector Array(WCDA) and Wide Field of view Cherenkov Telescope Array(WFCTA) and expected to be the highest sensitivity instruments in the gamma ray detection, ultra-high gamma survey, the primary cosmic ray spectra and anisotropy[1,2]. WFCTA, consisting of 18 telescopes, has been designed to fulfil the measurement of the primary cosmic rays with the energy of $10^{13}-10^{17} \mathrm{eV}$ and transfer the energy scales from the direct measurement to the extreme high energy measurement, with different lay-out in different observation mode and energy range[3, 4].

To meet the requirement calibration of WFCTA to select effectively the good shower events out and correct the raw data, improving the quality of the data and decreasing the systematic errors, it is primary importance to monitor the performance of laser devices in the calibration systems of LHAASO-WFCTA. Taking laser lidar to calibrate the ground-based high energy cosmic rays experiment is deployed by Auger collaboration[5, 6, 7, 8], Hires collaboration[9], The Telescope Array Collaboration[10] and so on.

The calibration system of the LHAASO-WFCTA consists of a series of devices, mainly including 2 YAG lasers, 2 nitrogen lasers, an infrared thermometer and a meteorological station. This calibration system is designed to study absolute calibration of photon number, air quality monitoring and weather, also study the measurement of the horizontal attenuation length, distribution of scattering angle, effective scaler height of atmospheric aerosols over LHAASO. Our collaboration had done cloud monitoring using Nitrogen laser[11].

\section{N2 laser device and YAG laser device}

\subsection{N2 laser system}

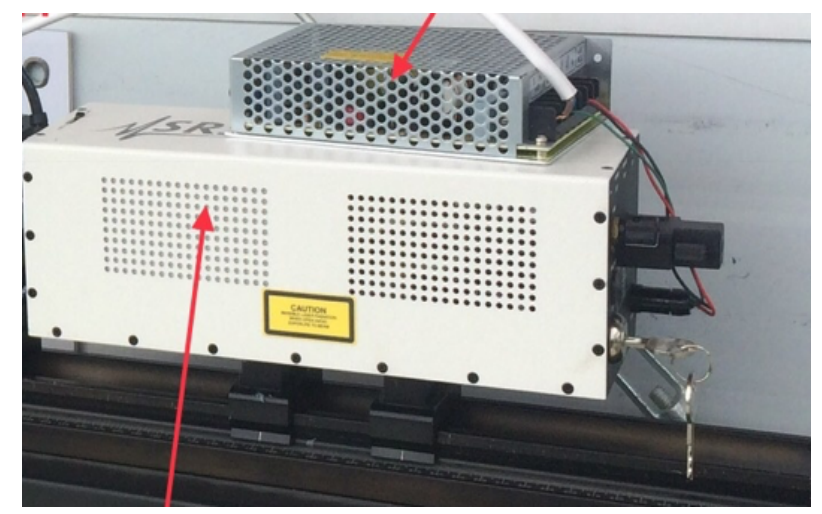

Figure 1: test layout of the NL100 N2 laser

The NL100 Nitrogen Laser device is small with the dimension of $27.9 \mathrm{~cm} \times 9.5 \mathrm{~cm} \times 9.5 \mathrm{~cm}$ as the lower arrow shown in Figure 1, a 24V direct power supplied the N2 laser as the upper arrow shown in Figure 1. The special parameters of the NL100 laser are listed in Table 1. The energy of 
laser pulse is measured by an energy meter, PE10BF-C made in Israel. The pulse repetition rate is chosen $1 \mathrm{~Hz}$ when the Laser devices run at LHAASO base.

Table 1: Specifications

\begin{tabular}{|c|c|c|}
\hline Parameteres & $\mathrm{N} 2$ & YAG \\
\hline Laser wavelength & $337.1 \mathrm{~nm}$ & $355 \mathrm{~nm}$ \\
Pulse repetition rate & $1-20 \mathrm{~Hz}$ & $1-20 \mathrm{~Hz}$ \\
Pulse width & $<3.5 \mathrm{~ns}(\mathrm{FWHM})$ & $7 \mathrm{~ns}$ \\
Pulse energy & $170 \mu \mathrm{J}$ & $100 \mu \mathrm{J}-2 \mathrm{~mJ}$ \\
Beam size & $3 \times 7 \mathrm{~mm}$ & $1.3 \mathrm{~mm}$ \\
Beam divergence & $5 \times 8 \mathrm{mrad}$ & $1 \mathrm{mrad}$ \\
\hline
\end{tabular}

\subsection{YAG laser system}

This laser system is mainly composed of a pulsed YAG solid-state laser device and a programmable logic controller (PLC).The PLC is able to achieve the control by local or remote PC. Water cooling is required while solid state laser device is operating. To ensure the laser device running normally in the lower temperature, that anti-frostbite liquid cycle system connecting to the inside of laser device. Also a protective device with thin quartz glass will be mounted on the laser device due to the windy and dusty environmen at LHAASO, shown in Figure 2. The glass has a angle of $\sim 5^{\circ}$ with the beam to prevent the damage of reflected light. The energy of laser pulse is also measured by the PE10BF-C.

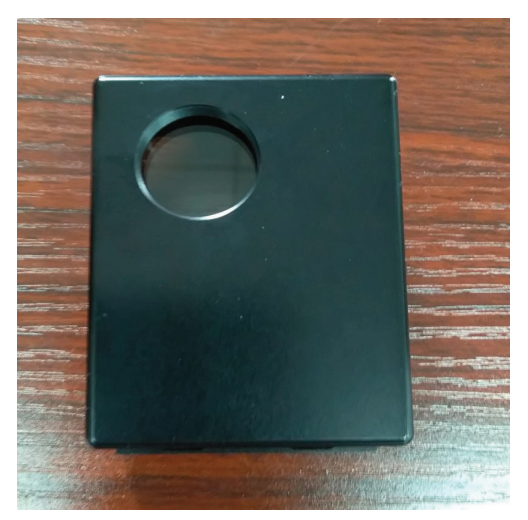

Figure 2: protective device for YAG device

The specifications of YAG laser are listed in Table 1.

\section{Performance of $\mathbf{N 2}$ laser and YAG laser}

\subsection{Performance of $\mathrm{N} 2$ laser}

Before the two NL100 Nitrogen lasers(name them NL100 1 and NL100 2) and YAG lasers began to run in LHAASO, their performance was monitored at Southwest Jiaotong University Campus and LHAASO base. When we tested the N2 laser or YAG laser, the PE10BF-C and laser 
device were put in a one-dimensional optical guide rail, and the PE10BF-C was $10 \mathrm{~cm}$ far away from the head of the device in order to observe the majority of energy of laser beam.

The NL100 laser quickly became stable after the power was supplied every time and they always ran stably in different temperature environgment. The Figure 3 shows one example of performance of the NL100 1 in Southwest Jiaotong University Campus, 0 km a.s.l.. The temperatue inside is $26.1^{\circ}$. The left pad of the Figure 2 illustrates the energy of the beam shot changes with the time and the right pad is the distribution of the energy. It is clear that the mean value of the laser beam is $163.8 \mu \mathrm{J}$ with the standard deviation of $4.868 \pm 0.078 \mu \mathrm{J}$.

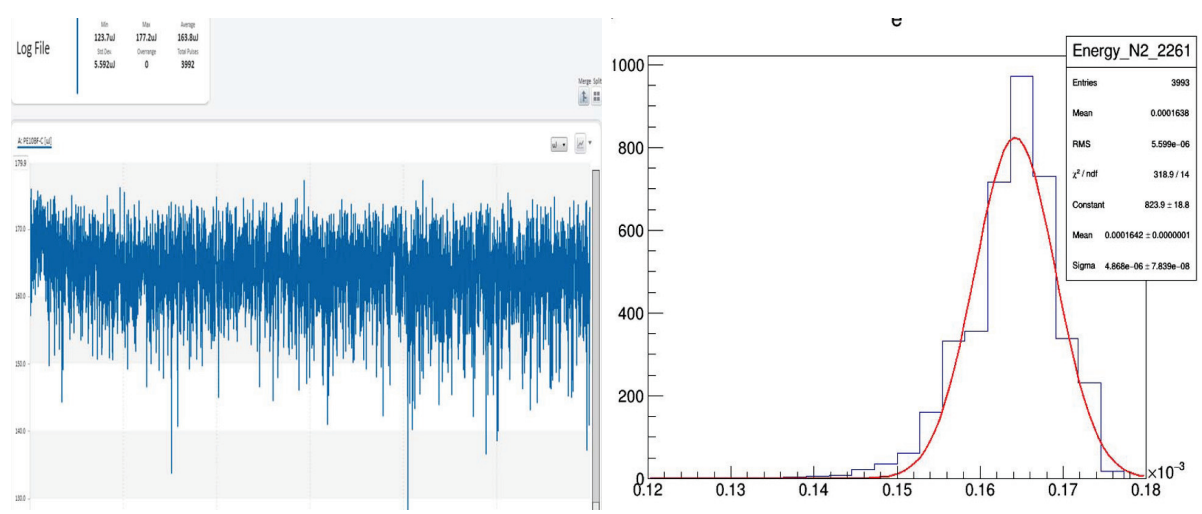

Figure 3: Performance in Southwest Jiaotong University Campus, Chengdu, 0km a.s.l, (left) Energy V.S. Time, (right) Distribution of the energy of laser beam

The Performance of 2 N2 laser devices in LHAASO base is presented in Figure 4. In the left pad, the laser device ran for one hour with the temperature inside of $12^{\circ}$. The mean value of energy of the NL100 2 laser beam is $165.5 \mu \mathrm{J}$ with the standard error of $5.558 \pm 0.071 \mu \mathrm{J}$. Similarly in the right pad, the laser device ran 25 minutes with the temperatue inside of $6^{\circ}$, the mean value of energy of the laser beam is $166.2 \mu \mathrm{J}$ with the standard error of $5.664 \pm 0.15 \mu \mathrm{J}$. According to the running at Southwest Jiaotong University Campus and LHAASO base, the energy of the NL100 2 Nitrogen lasers mostly assembled around $167 \mu \mathrm{J}$, and had no correlation with the environmental temperature.
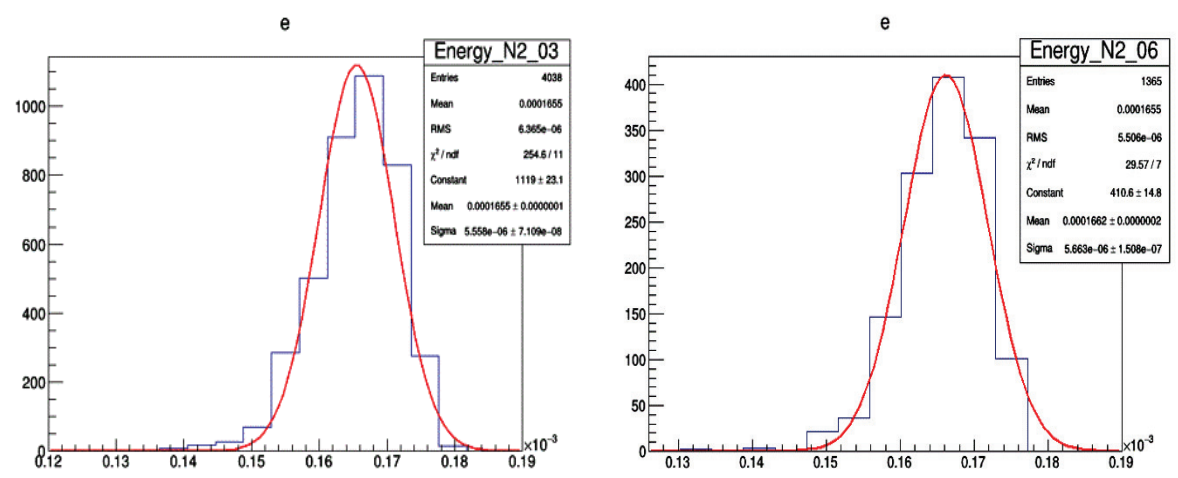

Figure 4: The distribution of the laser energy in LHAASO base, the unit of the energy is joule. left: $12^{\circ}$ of indoor temperature and running 1 hour, right: $6^{\circ}$ of indoor temperature and running 25 minutes 


\subsection{Performance of YAG laser}

Compared with NL 100 Nitrogen lasers, the YAG laser device is complex and difficult to operate. The ID of the two YAG laser devices are 1903070149,1903070249( we designate them L149 and L249). The energy of the laser beam change with the flash lamp voltage as shown in Figure 5, which indicates the YAG laser devices can work in different voltage of flash lamp. L149 has higher energy of laser beam than L249, but their energy can be reached to about $2 \mathrm{~mJ}$.

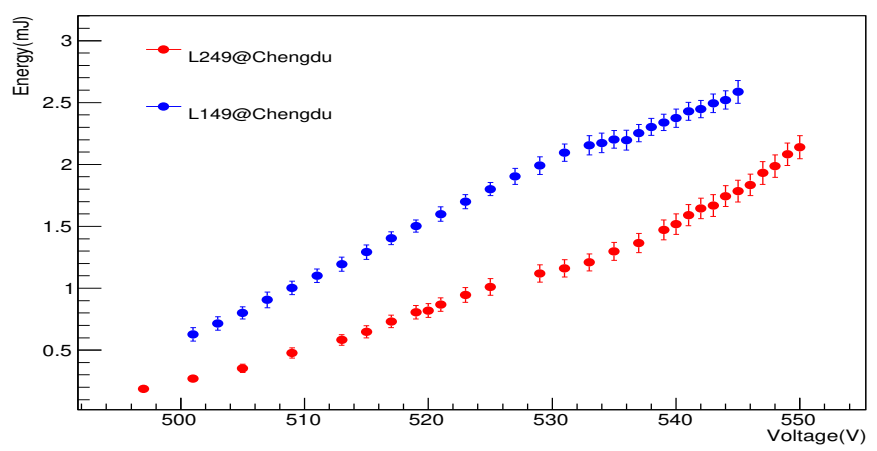

Figure 5: The energy of laser beam as a function of the voltage of flash lamp, blue color for L149, red color for L249
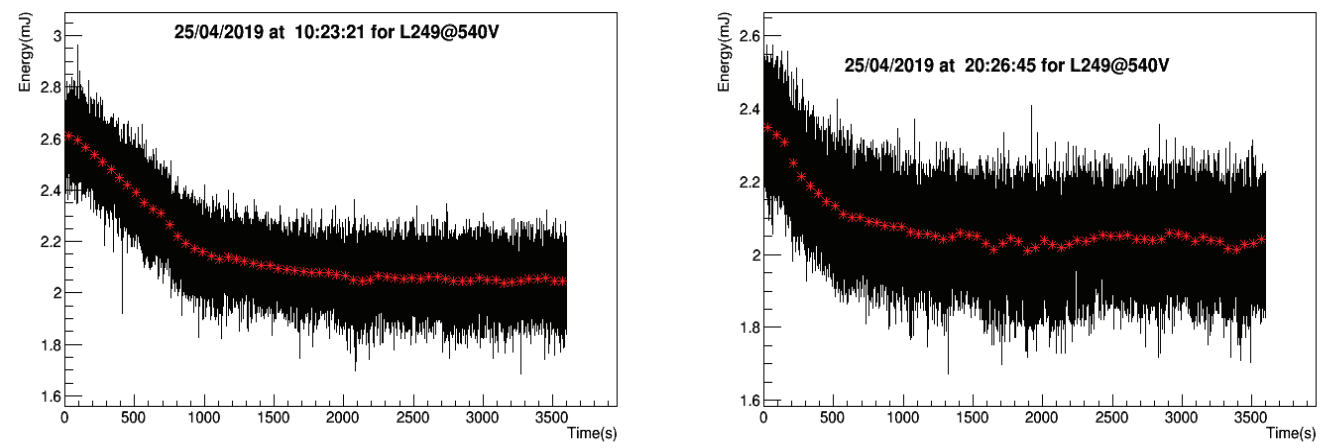

Figure 6: The performance of L249 in Southwest Jiaotong Univesity Campus, Chengdu( 0km a.s.l) left: L249 running 1hour from power supply in high indoor temperature, right: L249 running 1 hour from power supply in lower temperature. red color means the mean value in one minute.

The YAG laser usually take $25 \sim 30$ minutes to run stably after the power is on, which is indepent on the environmental temperature. Howerer, the energy of laser beam will became smaller if the environmetal temperature decrease like shown in the Figure 6. On the morning of April 25, 2019, the temperature is higher than the night, the energy of the L249 laser pulse showed a little smaller. Compaired with L249, the temperature has a significant effect on the energy of laser pulse of L149. Also the L149 has ability to record the weak effect from envrionment, as shown in the little peak in about 2000s in left pad of Figure7.

It is the first time the YAG laser tried to run in the altitude with bigger than $3000 \mathrm{~m}$. Maybe the thin air at LHAASO site, the YAG laser will take much fewer time to turn stable, as shown in 

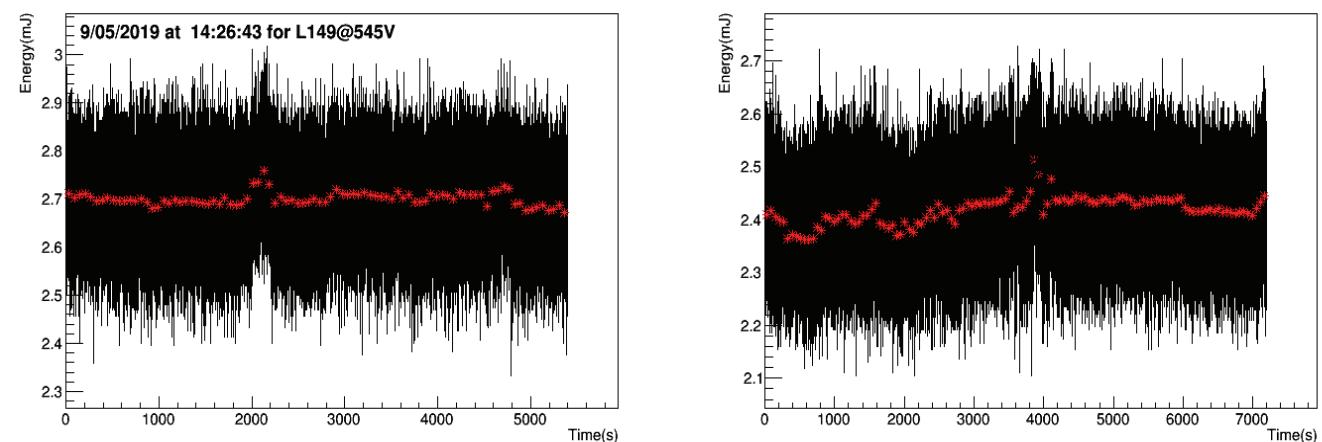

Figure 7: The performance of L149 in Southwest Jiaotong Univesity Campus, Chengdu( 0km a.s.l) left: L149 running in stable status in higher indoor temperature, right:L149 running in stable status in lower indoor temperature, red color means the mean value in one minute.

Figure 8. The red points are the mean values of the laser pulse in one minute. It took less than 10minutes to make the YAG laser in stable running like in the Figure 8(upper left). The energy of the stable laser pulse is $1.875 \mathrm{~mJ}$ with standard error $0.071 \mathrm{~mJ}$ when the voltage of flash lamp was set to $535 \mathrm{~V}$ in the indoor temperature, which meet the requirement of the calibration of LHAASOWFCTA. Similarly the environgmental temperature affect the energy of the laser pulse as show $\mathrm{n}$ in the middle(right) pad of Figure 8. The energy became smaller mostly because of the lower temperature after 2500s at the deep night of LHAASO. The Laser 249 also has similar performance with L149 and succeded to run in LHAASO base as shown in the lower pads of Figure 8. The L249 ran stably within 20 minutes on the noon of May 27, 2019 with the mean energy $1.885 \pm 0.0677 \mathrm{~mJ}$.

\section{Summary}

After testing of 2 N2 laser system and 2 YAG laser system in the plain and plateau, separately, their good performance have been confirmed. The $2 \mathrm{~N} 2$ laser system operate simply and the energy pulse is alway stable if the power is supplied. The stable energy is not dependent on the environmental temperature and altitude. It takes some time to make the 2 YAG laser systems in stable status both in plain and plateau. This laser pulses is significantly dependent on the environmental temperature and altitudes, also need to run long time to observe their stablitiy in futher.

\section{References}

[1] Z. Cao, J. L. Liu, Y. X. Bai, Chinese Journal of Nature, 31(6): 342-347 (2009)(in Chinese)

[2] Z. Cao, Chinese Physics C, 34(2): 249-252 (2010)

[3] S. S. Zhang, Y. X. Bai, Z. Cao, et al, Nuclear Instruments \& Methods in Physics Research Section A-accelerators Spectrometers Detectors and Associated Equipment, 629(1): $57-65$ (2011)

[4] H. C. Li, M. J. Chen, H. Y. Jia, et al, Chinese Physics C, 1: 49-55 (2014)

[5] B. Fick, et al, JINST 1, P11003(2006)

[6] S.Y. BenZvi, et al, Nucl. Instrum. Meth. A574,171(2007) 

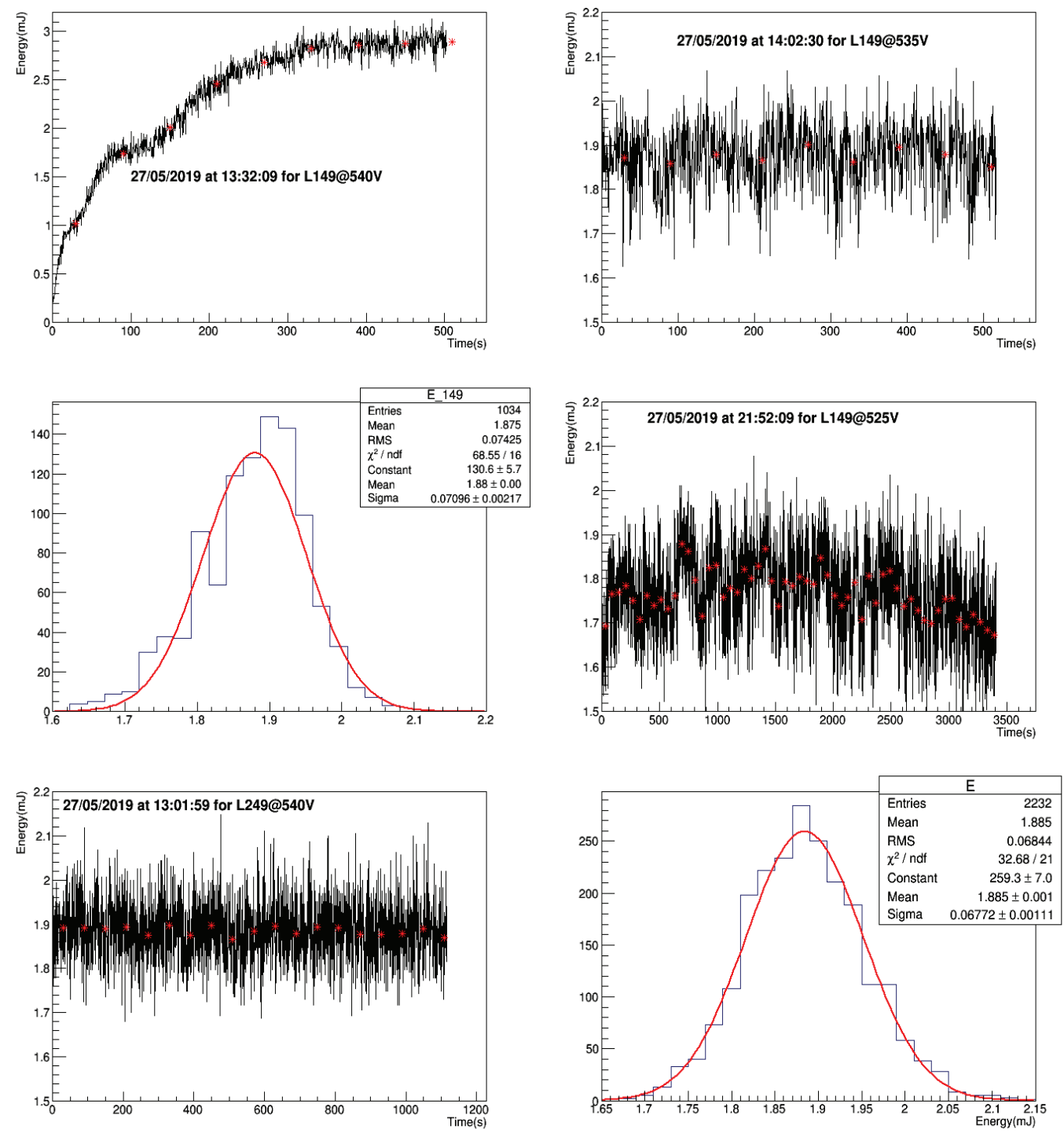

Figure 8: The YAG laser L249 and L149 ran successfully at LHAASO

[7] S.Y. BenZvi, et al, Astropart. Phys. 28, 312(2007)

[8] S.Y. BenZvi, et al, Proc. 30th ICRC, Vol 4, 347,2007

[9] R.U. Abbasi, et al., Astropart. Phys. 25, 74(2006)

[10] The Telescope Array Collaboration, Nucl. Instrum. Meth. A597,61(2008)

[11] Z.D. Sun, Y. Zhang, F.R. Zhu et al, ICRC 2015, 641 